\title{
Dealing with a 'hidden stressor': emotional disclosure as a coping strategy to overcome the negative effects of motive incongruence on health
}

\author{
Julia Schüler ${ }^{1, *, \dagger}$, Veronika Job ${ }^{1}$, Stephanie M. Fröhlich ${ }^{2}$ and Veronika Brandstätter ${ }^{1}$ \\ ${ }^{1}$ University of Zürich, Switzerland \\ ${ }^{2}$ University of Osnabrück, Germany
}

\begin{abstract}
Summary
Taking the affiliation motive as an example, present research examines whether the negative effects of implicit-explicit motive incongruence on health is moderated by emotional disclosure. Starting from the point of view that motive incongruence works as a chronic stressor and therefore causes impairment of health, we predicted that participants who use the stress-reducing coping strategy of emotional disclosure should be less affected by the negative effects of motive incongruence on health than participants who do not use this stress-coping strategy. Two studies confirmed this hypothesis. Participants with affiliation motive incongruence who practiced emotional disclosure used less medication (Study 1, $n=85$ ) and reported lower somatization symptoms (Study 2, $n=$ 102) than motive incongruent individuals who did not disclose their emotions to others. Copyright
\end{abstract}

\section{Key Words}

motive incongruence; emotional disclosure; health; stress; affiliation motive

\section{Introduction}

Stressful life events and traumatic experiences can impair subjective well-being and health. There is a growing body of empirical data suggesting that also non-traumatic general life stress as,

\footnotetext{
*Correspondence to: Julia Schüler, University of Zürich, Department of Psychology, Psychology of Motivation, Volition and Emotion, Binzmühlestrasse 14/6, 8050 Zürich, Switzerland

${ }^{\dagger}$ E-mail: j.schueler@psychologie.uzh.ch
}

for example, daily, occupational stressors (e.g. Dormann \& Zapf, 2004; Story \& Repetti, 2006), worrying about one's financial situation and health (Grulke et al., 2006), time pressure (Roxburgh, 2004) and life transitions stressors (e.g. adjustment to college, residential independence; Kiselica, Baker, Thomas, \& Reedy, 1994; Lee \& Gramotnev, 2007) has negative effects on subjective and physiological wellbeing.

In the present research we suggest motive incongruence to be an important source of general life stress. Many studies showed that the 
incongruence between the affective-based implicit motive and the cognitive-based explicit motive (Baumann, Kaschel \& Kuhl, 2005; Hofer \& Chasiotis, 2003; Hofer, Chasiotis, \& Campos, 2006), or between the implicit motive and goals (Brunstein, Schultheiss, \& Grässmann, 1995; Kehr, 2004; Schultheiss \& Brunstein, 1999), have negative effects on subjective as well as physiological well-being. The negative effects are explained by suggesting that these types of incongruence produce enduring intrapersonal conflicts, which work as chronic stressors (e.g. Baumann et al., 2005) and thereby impair subjective wellbeing and health. In order to examine this explanation, we tested the following assumption: if incongruence leads to chronic stress, which again impairs well-being and health, then individuals who apply an adequate strategy to cope with stress should report less impairment of well-being and health compared to individuals who do not use a stress-coping strategy, even though both groups have incongruent implicit and explicit motives or goals. In the following paragraphs we will first theoretically and empirically embed our assumption of incongruence as a chronic stressor and will then introduce emotional disclosure as an adequate stress-coping strategy.

\section{Motive incongruence as a 'hidden stressor'}

Research on motive incongruence and well-being roots in the distinction of two separate motivational systems, implicit motives and selfattributed motives (also called explicit motives, DeCharms, Morrison, Reitman, \& McClelland, 1955). The authors assessed individuals' achievement motive from stories, which were written to pictures. They found that the motive disposition was uncorrelated with the self-reported desire for achievement. From this the authors concluded that the motivational system can be separated into the implicit and explicit motivational system. Empirical and theoretical evidence for this separation was summarized in an article by McClelland, Koestner and Weinberger (1989), stating that implicit motives are based on affects and are not consciously represented, whereas the self-reported motives are cognitive evaluations about the self. Further, implicit motives and self-attributed motives influence different classes of behaviour, indicating that implicit motives are associated with spontaneous behavioural trends over time, whereas explicit motives predict immediate responses to specific situations that are often based on cognitive decisions. Whereas implicit motives are aroused by implicit incentives, for example, experiences in the course of action itself, explicit motives are elicited by explicit incentives such as social expectations or demands (cf. McClelland et al., 1989). Implicit motives are developed by emotional experiences early in life, whereas explicit motives are self-attributes, which are shaped by the influence of environmental expectancies and demands. In addition to the explicit motives, also goals belong to the explicit motivational system. They are defined as 'internal representations of desired future states' (Schultheiss \& Brunstein, 1999, p. 3) and share the basic characteristics distinguishing explicit from implicit motives, as, for example, the cognitive basis and the conscious act of self-attribution.

It has to be noted that the two separate motivational systems (implicit vs. explicit) can either be congruent or incongruent. In a congruent motivational system the motives form a coalition and work together to generate and direct behaviour. Incongruence is given when an implicit motive lacks a corresponding explicit motive (or goal), or vice versa. McClelland and colleagues postulated that the incongruence 'between implicit and explicit motives [...] certainly leads to trouble' (McClelland et al., 1989, p. 700). In recent years, empirical evidence has grown confirming the predicted trouble by showing that the incongruence between implicit motives and explicit motives as well as between implicit motives and goals is associated with impairment of emotional well-being (e.g. Brunstein et al., 1998; Brunstein, Schultheiss, \& Maier, 1999; Brunstein, Lautenschläger, Nawroth, Pöhlmann, \& Schultheiss, 1995), life satisfaction (Hofer \& Chasiotis, 2003; Hofer et al., 2006) and decreased physiological well-being (Baumann et al., 2005) (for the negative effects of incongruent motives in a broader sense see also Kasser \& Ryan, 1996; Ryan, et al., 1999; Sheldon \& Elliot, 1999; Sheldon \& Kasser, 1995).

The explanations for the impairment of well-being due to motive incongruence have in common that they all assume that incongruence causes a kind of intrapersonal conflict or stress. For example, Brunstein et al. (1998) argued (referring to McClelland, 1985) that individuals feel happy and pleased when they engage in 
behaviour that is in accordance with their implicit motives. Vice versa, not engaging in behaviour that matches the implicit motive puts the implicit motive under stress. Because explicit motives or goals transform implicit motives into concrete motive-satisfying behaviour (Brunstein et al., 1998; Elliot, Gable, \& Mapes, 2006), the lack of a corresponding explicit motive or goal 'stresses' the implicit motive. This stressed motive again results in dissatisfaction (see also McClelland \& Jemmott, 1980; McAdams \& Bryant, 1987). In accordance to their assumptions, Brunstein and colleagues found that progress towards goals only lead to emotional well-being when the goals were congruent with the implicit motives but not when they were incongruent (Brunstein et al., 1998).

Also Kehr (2004) assumed that intrapersonal conflicts are responsible for impairment of subjective well-being. The author argued that the incongruence between implicit and explicit motives leads to incompatible behavioural tendencies that cause psychological conflicts within a person. These intrapersonal conflicts consume volitional strength, which in the long run impairs subjective well-being. Kehr (2004) tested this hypothesis among managers and found his hypothesis confirmed: The influence of implicit and explicit motive incongruence on impaired subjective well-being was mediated by a decrease in volitional strength that resulted from intrapersonal conflicts.

Also Baumann et al. (2005) assumed that intrapersonal conflicts caused by motive incongruence can impair subjective-well-being and even form physical symptoms. Although the authors were mainly interested in scrutinizing whether stressful life events reduce motive congruence when the ability to self-regulate affect was impaired, they made important assumptions relevant for our research question. Focusing on the achievement motive, they supposed that the incongruence between implicit and explicit achievement motives is a hidden source of stress ('bidden stressor', p. 783). They used the term hidden because individuals are not aware of their motive incongruence. The authors suggested that a strong explicit achievement motive, which is not based on a corresponding implicit motive and therefore lacks its energizing function, 'leads to accumulation of conflict, frustrates basic needs, and increases cortisol concentration' (Baumann et al., 2005 , p. 291, see also Winter, 1996). Due to this lacking explicit achievement motive a high implicit motive can not be transformed into behaviour, which again facilitates the emergence of physical symptoms. Because motive incongruence is a relatively stable disposition, the intrapersonal conflicts and the increased cortisol concentration become chronic. A high cortisol level over a long time is known to be associated with a variety of somatic symptoms (e.g. Sapolski, 1992). The empirical data of Baumann et al. (2005) supported this assumption by revealing achievement motive incongruence as a predictor of the course of psychosomatic complaints (e.g. head-ache, stomach-ache, back-pain) among a clinical population over a time period of 3 months. In the present research we borrow the term 'hidden stressor' from Baumann et al. (2005) in order to metaphorically express the unconscious character of the stressor. Additionally, we want to emphasize the chronic character of this hidden source of stress by calling it a chronic hidden stressor.

To sum up the explanations that researchers provided for the negative effects of the incongruence between implicit motives and explicit motives or goals, the incongruence is assumed to produce intrapersonal chronic stress that in the long run impairs subjective well-being and health.

\section{Emotional disclosure as a stress-coping strategy}

A good way to cope with intrapersonal stress is emotional disclosure. There is broad empirical evidence showing that expressing stress-related emotions and thoughts improve health and subjective well-being (Francis \& Pennebaker, 1992; Lepore \& Smyth, 2002; Pennebaker \& Beall, 1986; Pennebaker, 1997; Smyth, 1998; Frattaroli, 2006). For example, emotional expression was found to be associated with enhanced immune functioning (Petrie, Booth, Pennebaker, Davidson, \& Thomas, 1995; Lepore \& Smyth, 2002), less physician visits (e.g. Lepore \& Greenberg, 2002), lower systolic and diastolic blood pressure (McGuire, Greenberg, \& Gevirtz, 2005), increased positive affect (e.g. Mendolia \& Kleck, 1993), decreased distress (Lepore, 1997) and improved psychological health (e.g. Sloan \& Marx, 2004).

The positive effects of emotional disclosure can be approached by writing about (Park \& 
Blumberg, 2002; Pennebaker, 1997; Pennebaker \& Beall, 1986) or talking about stressful events (e.g. Murray \& Segal, 1994; Parkinson \& Totterdell, 1999; Rimé, Philippot, Boca, \& Mesquita, 1992). Pennebaker and his colleagues convincingly demonstrated the effectiveness of a writing paradigm in which participants were encouraged to write 'about [their] very deepest thoughts and feelings about an extremely important emotional issue that has affected [them] and [their] life' (Pennebaker, Zech, \& Rimé, 2001, p. 523). The writing paradigm worked for different populations (e.g. children, elderly people, students, maximum security prisoners) and across a variety of dependent measures, for example, health, self reported psychological well-being and behavioural changes (Park \& Blumberg, 2002; Pennebaker, 1997; Smyth, 1998). Emotional expression through talking with others was examined by Rimé (e.g. Rimé, 1987, 1999; Murray \& Segal, 1994; Parkinson \& Totterdell, 1999). He and his colleagues observed that people have the strong urge to talk about stressful or other emotional experiences and that individuals report that talking helps them to deal with stressful events (for a review, see Rimé et al., 1992).

The mechanism behind these positive effects assumedly lies in the disclosure of emotions. This disclosure changes the emotional and cognitive processing of stressful experience in a way that the stressful experiences lose their power to distract cognition, disrupt emotions and to trigger physiological stress reactions (Lepore, Greenberg, Bruno, \& Smyth, 2002; Pennebaker, Mayne, \& Francis, 1997). The cognitive changes as a result of emotional disclosure may include that individuals gain a deeper understanding of their negative emotional responses to stressors (Lepore et al., 2002), which again facilitates emotionregulation strategies (Greenberg, Wortmann, \& Stone, 1996). According to Pennebaker and Seagal (1999), putting stressful experiences into words through emotional disclosure fosters the integration of those experiences into a coherent narrative that may render the stressful experience more meaningfully. Once a person has developed a new meaning of the stressful experience, the event can 'be summarized, stored, and forgotten more efficiently' (Pennabaker \& Seagal, 1999, p. 1248).

Research on emotional disclosure suggests that coping with stress using emotional disclosure is especially effective for individuals who are exposed to a high degree of any kind of stress (e.g. life stress, trauma). Thus, it works well for students who struggle to get adjusted to college (Pennebaker, Colder, \& Sharp, 1990; Pennebaker \& Francis, 1996), for individuals high in fear of failure (Langens \& Schüler, 2005), for homeless people (deVicente, Munoz, Perez, \& Santos-Olmo, 2004) and for many clinical populations, as for example, patients with post-traumatic stress disorder (e.g. Bernard, Jackson, \& Jones, 2006; Sloan, Marx, \& Epstein, 2005), roimatoid arthritis (Kelley, Lumley, \& Leisen, 1997), chronic pain (Norman, Lumley, Dooley, \& Diamond, 2004) or cancer (Zakowski, Ramati, Morton, Johnston, \& Flanigan, 2004).

Taken together, previous research showed that emotional disclosure has strong positive effects on subjective well-being and health. This seems especially valid for individuals who are exposed to a high degree of stressful experiences.

In the present research we integrated research on motive incongruence and emotional disclosure. We started from the point of view that motive incongruence works as a 'hidden stressor' that again decreases immune function and thereby in the long impairs health (see Baumann et al., 2005). We continued our line of argumentation by assuming that when the chronically hidden stressor is the reason why motive incongruence is associated with health impairment, individuals who are able to cope with stressful events by applying an effective coping strategy should report a low impairment of health despite their motive incongruence. Disclosing stressful life events to others has proven to be an effective strategy to cope with stress (see Smyth, 1998). Integrating the research of motive incongruence and emotional disclosure, we assume that emotional disclosure reduces the negative effects of stress caused by motive incongruence. Specifically, we hypothesize that emotional disclosure moderates the relationship between motive incongruence and health. Motive incongruent individuals who disclose their emotions to others are assumed to report less health impairment than motive incongruent individuals who do not disclose their emotions. For motive congruent individuals who do not experience many stressful experiences, we assumed no differences whether or not they use the conflict-coping method of emotional disclosure. 


\section{The Present Research}

In order to put our hypothesis into empirical practice, we chose talking (instead of writing) about stressful experiences as an emotional disclosure measure, and focused on the incongruence of implicit and explicit affiliation motives. The reason for using talking instead of writing was that we wanted to examine the natural and most common way of dealing with everyday life stressors. Across different studies, Rimé found that in 90 per cent of the cases emotional experiences were verbally disclosed to others (for a review, see Rimé et al., 1992).

We focused on the affiliation motive, because the 'hidden stressor' hypothesis has only been analysed for the achievement motive so far (Baumann et al., 2005), and we wanted to demonstrate its generalizability to other motives. The affiliation motive is defined as the desire to 'form friendship and association. To greet, join and live with others. To cooperate and converse sociably with others. To love, to join groups' (Murray, 1938, p. 83). Potential goals for the affiliation motives are, for example, being in the company of others, being friends with others and exchanging views. To reach those goals, affiliation-motivated individuals show affiliation motive-related activities, such as getting to know people, showing goodwill and affection, and pleasing others (see Baumeister \& Leary, 1995; Sokolowski, 2008).

In order to demonstrate the robustness of the assumed moderator effect, we measured the incongruence between the implicit and explicit motivational system using different methods. In Study 1 we measured the incongruence between implicit motives and goals. Study 2 addressed the incongruence between implicit motives and explicit motives. Additionally, we varied the measures of emotional disclosure and health.

\section{Study 1}

The explicit motivational system is cognitively represented and therefore can be measured using individuals self-reports about their motives and goals. This can be done by asking participants for their current goals (Brunstein, 1993; Brunstein et al., 1995) or by applying questionnaires measuring individuals' preferences for situations (e.g. Personality Research Form, PRF; Jackson, 1984) or values (e.g. Personality Value Questionnaire, PVQ; McClelland, 1991). In contrast, implicit motives are unconscious and therefore can only be measured by using projective tests like the Thematic Apperception Test proposed by Murray (1943), or with tests based on the principle of projection as the Multi-Motive-Grid (MMG; Sokolowski, Schmalt, Langens, \& Puca, 2000) or the Operant Multimotive Test (OMT; Kuhl \& Scheffer, 1999). In the present study we used the TAT to measure the implicit affiliation motive and a goal measure to assess the explicit affiliation motive. In order to assess emotional disclosure we referred to results gained by Panagopoulou, Maes, Rimé and Montgomery (2006) showing that the quality of emotional disclosure, rather than its quantity, was negatively related to distress caused by a stressful experience. Therefore we measured the quality of emotional disclosure by asking participants for their frankness and honesty while disclosing emotions to other persons. As an indicator for health we used the amount of medicine intake.

\section{Method}

\section{Participants and procedure}

Participants were 60 female and 25 male undergraduate students with an average age of 23 years [standard deviation $(\mathrm{SD})=4.92$ ]. They filled in the measures of implicit motives, goals and health in a group session and answered the emotional disclosure questionnaire at home.

\section{Measurements}

Explicit affiliation motive. To measure the affiliation goal we followed a procedure used by Brunstein et al. (1998). Participants were asked to write down the two most important affiliation goals for which they are currently striving. Afterwards, participants rated their goals concerning ten items on a scale ranging from 1 (disagree strongly) to 5 (agree strongly). Four items measured commitment to the goal (e.g. I can hardly wait to start working on this goal) and six items measured the goals attainability (e.g. I have many opportunities in my everyday life to work on this goal). The average commitment 
for both goals was $3.81(\mathrm{SD}=0.57)$ and their mean attainability was $3.1(\mathrm{SD}=0.49)$. Commitment and attainability were significantly correlated $(r=0.29, p<0.01)$. Referring to the basic assumption of the expectancy-value approach on motivation (e.g. Atkinson, 1957; see Heckhausen, 1989), we multiplied the $z$-transformed value for expectancy to reach the affiliation goal (attainability) with the $z$-transformed value of that goal (commitment) in order to get an index for the explicit affiliation motive strength $(M=0.28$; $\mathrm{SD}=1.14)$

Implicit affiliation motive. Participants invented fictive stories to four TAT pictures displaying a man sitting at a desk, a ship's captain talking with another man, two female scientists in a laboratory and a man and a woman on a trapeze (Smith, 1992). The scoring for affiliation motive imagery was carried out by two independent scorers who attained sufficient reliability across several previous studies. They used Winter's (1991) Manual for Scoring Motive Imagery in Running Text and agreed with a Kappa of 0.81. The scoring disagreements were discussed in an additional session and a second independent rating revealed a Kappa of 0.95. Due to a significant correlation between affiliation motive raw scores and the length of the written stories $(r$ $=0.26, p<0.01)$, the scores were corrected by regression for story length $(M=0.14, \mathrm{SD}=1.59)$ (Smith, Feld, \& Franz, 1992).

Affiliation motive incongruence. In order to get an affiliation motive incongruence index, we computed the absolute difference of standardized implicit and explicit affiliation motive scores. The higher the score, the larger is the incongruence between the implicit and the explicit motive. This procedure has proved useful in motiveincongruence research (e.g. Baumann et al. 2005; Kehr, 2004).

Emotional disclosure. To assess the quality of participants' engagement in emotional disclosure we used a modified version of the selfdisclosure questionnaire (SESI; in German: Selbstenthüllungstest; Göhring, 2002). It comprises 16 different themes where individuals typically emotionally disclose themselves as for example 'people I'm afraid of' and 'happy moments in my life'. For each of these 16 items participants rated how frankly and honestly they had talked about those topics to another person. They rated these items on a three-point scale ranging from 0 (not honest at all) to 2 (very bonest). An index of the overall quality of emotional disclosure was created by computing the average honesty across the 16 items.

Health. To measure physical well-being, participants were asked about their medicine intake (analgetic drugs, tranquillizing drugs, stimulating pharmaceuticals, others). For each type of medicine they rated the frequency of intake during the last few weeks using a five-point scale ranging from 0 (never) to 4 (several times a week). Their scores were summed up in an index of general medicine intake.

\section{Results and brief discussion}

\section{Preliminary analyses and descriptive statistics}

Exploratory analyses showed that neither gender nor age of participants had significant impact on the results reported below. The correlation between motive incongruence $(M=0.99, \mathrm{SD}=$ $0.87)$ and quality of emotional disclosure $(M=$ $1.71, \mathrm{SD}=0.17)$ was $r=0.02$. Affiliation motive incongruence and emotional disclosure were both marginally significantly associated with medicine intake $(M=1.43, S D=2.08)$ (incongruence: $r=$ $0.20, p<0.10$; emotional disclosure: $r=0.21, p$ $<0.10)$.

\section{The working together of motive incongruence and emotional disclosure}

A hierarchical regression analysis was conducted with affiliation motive incongruence and emotional disclosure ${ }^{1}$ employed in Step 1 of the regression equation. In Step 2 the motive incongruence $\times$ emotional disclosure-interaction was introduced. Table I shows that motive incongruence was a significant predictor of medicine intake, $b=0.24, s e_{\mathrm{b}}=0.10, t(81)=2.43, p<0.05$. Emotional disclosure was negatively related to medicine intake, $\left(b=-0.21, s e_{\mathrm{b}}=0.10, t(81)=\right.$ $-2.17, p<0.05)$. Additionally, the interaction of

\footnotetext{
${ }^{1}$ Continuous variables were centered before entering them into the hierarchical regression analyses (Cohen, Cohen, West, \& Aiken, 2003).
} 
Table I. Hierarchical regression of medicine intake (Study 1).

\begin{tabular}{llcccc}
\hline Step & Variable & $\Delta R^{2}$ & $d f$ & $\Delta F$ & $b^{\mathrm{a}}$ \\
\hline 1 & Main effects & 0.09 & 2,82 & $3.85^{*}$ & \\
$\quad \begin{array}{l}\text { Motive incongruence } \\
\quad \text { Emotional disclosure }\end{array}$ & & & & $0.24^{*}$ \\
2 & $\begin{array}{l}\text { Motive incongruence } \times \text { emotional } \\
\text { disclosure }\end{array}$ & 0.13 & 1,81 & $12.93^{* *}$ & $-0.26^{* *}$ \\
& Cumulative $R^{2}$ & 0.21 & 3,81 & $7.25^{* *}$ & \\
\hline
\end{tabular}

Note: ${ }^{\mathrm{a}} b$ is the standardized regression coefficient in the regression equation.

" $p<0.05 ;$;" $p<0.01$.

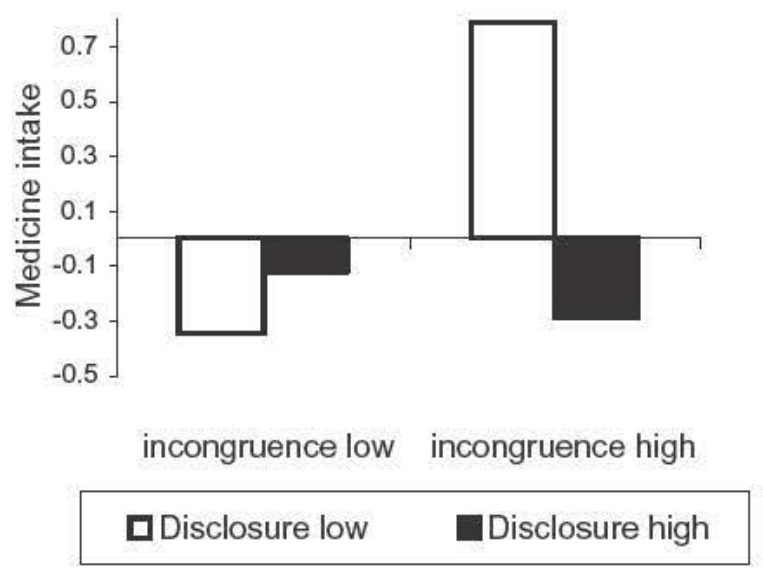

Figure 1. Medicine intake as a function of motive incongruence and emotional disclosure (Study 1).

motive incongruence and emotional disclosure predicted medicine intake $\left(b=-0.32, s e_{\mathrm{b}}=0.09\right.$, $t(83)=-3.60, p<0.01)$. The interaction effect is illustrated in Figure 1 (following the procedure by Cohen, Cohen, West, \& Aiken, 2003), indicating that participants with a high incongruence between their implicit and explicit affiliation motives $^{1}$ who are engaged in qualitatively good emotional disclosure consumed less medicine than participants with a high incongruence and who did not engage in emotional disclosure. Post hoc analyses (Johnson-Neyman technique) showed that the quality of emotional disclosure made a difference for participants with a high motive incongruence $\left(b=-0.54, s e_{\mathrm{b}}=0.13, t(81)\right.$ $=-4.03, p<0.01$, two-tailed test), but made no difference for participants with a low motive incongruence $\left(b=-.11, s e_{\mathrm{b}}=0.13, t(81)=0.82\right.$, ns, two-tailed test).

\section{Brief discussion}

Study 1 confirmed the expected moderator effect showing that the effect of affiliation motive incongruence on medicine intake was buffered by qualitatively good emotional disclosure. In order to demonstrate that the moderator effect also holds true for another form of incongruence, Study 2 measured the implicit affiliation motive and the explicit affiliation motive using different measures. Additionally, by using a bigger sample size we hoped to enhance confidence in the effects.

\section{Study 2}

Study 2 was designed to replicate the moderator function of emotional disclosure in the interrelationship of motive incongruence and health. In order to demonstrate that the hypothesized moderator effect is independent of the way how the central variables are measured, Study 2 used the Operant Multimotive Test (OMT; Kuhl, \& Scheffer, 1999) as the implicit affiliation motive measure. The Personality Value Questionnaire (PVQ; McClelland, 1991) was used to assess the explicit affiliation motive.

Research of Rimé and colleagues (e.g. Rimé, 1987,1999 ) shows that individuals often choose spouses or family members as recipients for emotional disclosure, because of their close attachment to them (Rimé et al., 1992). Therefore we operationalized emotional disclosure by administering a questionnaire of dyadic coping (Bodenmann, 1990). This questionnaire focuses on emotional disclosure within intimate relationships and contains important facets of emotional disclosure, as for example, talking about the emotional impact of stressful events. Similar to the 
study conducted by Baumann et al. (2005), participants of the present study were asked for their somatic complaints.

\section{Method}

\section{Participants and procedure}

One hundred and two participants (51 females) with a mean age of 26.1 years were recruited through flyers and verbal propaganda at the University of Osnabrück, Germany. Participants received a questionnaire booklet at the University of Osnabrück and were asked to return the completed questionnaires within the next days. After handing over the booklet they either got paid 15 EURO or course credits for their participation.

\section{Measurements}

Explicit affiliation motive. We used the affiliation scale of the Personal Values (McClelland, 1991) questionnaire to measure the self-reported affiliation motive. The 10 items had to be evaluated according to their personal importance along a scale from 0 (not important for me) to 6 (exceedingly important for me) (e.g. 'having plenty of time to spent with my friends or family', 'close, friendly, cooperative relations with others') and were summed up ( $M=38.04$; $S D=5.56$; Cronbach's Alpha in present study = $0.76)$.

Implicit affiliation motive. The Operant Multimotive Test (OMT; Kuhl \& Scheffer, 1999) measured the implicit affiliation motive. Using a modified TAT technique, 15 pictures were shown and the participants were asked to invent a story (without having to write down the story) and give their spontaneous associations to the following questions: (1) 'What is important for the person in this situation and what is the person doing?' (2) 'How does the person feel?' (3) 'Why does the person feel this way?' (4) 'How does the story end?' The OMT differentiates five components for the implicit affiliation, achievement, and power motive. The affiliation motive is made up of (a) sharing emotions; (b) having fun with others; (c) coping with rejection, and working on relations; (d) desire for or feeling of security; and (e) fear of rejection and being alone. For the purpose of the present studies, the sum of the five components was computed to assess the implicit affiliation motive $(M=2.97 ; \mathrm{SD}=1.46)$. The OMT has a sufficient internal consistency, even according to indices based on classical test theory: $\alpha=0.74$ and sufficient retest stability of $r=0.72$ (Scheffer, 2005; Scheffer, Kuhl, \& Eichstaedt, 2003). The scoring was carried out by well-trained assistants who had reached sufficient reliability across several studies.

Affiliation motive incongruence. The affiliation motive incongruence index was calculated in the same way as in Study 1. We computed the absolute difference of standardized implicit and explicit affiliation motive scores which was standardized. The higher the score, the larger is the incongruence between the implicit and the explicit motive.

Emotional disclosure. The Stress Communication Scale from the Fragebogen zur Erfassung des Dyadischen Copings als Tendenz (English translation: Questionnaire to assess dyadic coping as a tendency; FDCT-N; Bodenmann, 1990) was administered to quantify emotional disclosure. The Stress Communication Scale consists of four items (Cronbach's Alpha in this study $=0.66$ ), which had to be rated on a scale ranging from 0 (never) to 4 (very often). One example item is 'I am frank with my partner, telling him when I am stressed and when I need his emotional support'.

Health was measured with the somatization scale from the Symptom Checklist (SCL; Franke, 1994). The three somatization items (numbness or tingle in parts of the body, feeling of faintness in parts of the body, feelings of heaviness in arms or legs) were rated on a scale from 1 (not at all) to 4 (very strong), answering the question: 'How strong did you suffer from [...] in the last seven days?' Cronbach's Alpha in this study was 0.70 .

\section{Results and brief discussion}

\section{Descriptive statistics and intercorrelation of variables}

Correlation analyses (Pearson; two-tailed) showed that neither motive incongruence $(M=1.06$, $\mathrm{SD}=0.78)$ nor emotional disclosure $(M=7.00$, $\mathrm{SD}=1.35)$ were significantly correlated with the somatization scale $(M=1.13, \mathrm{SD}=1.57)$, 
$r=0.04$ and -0.07 , respectively. Women had a significant higher score in emotional disclosure $(M=7.53 ; S D=1.26)$ than men $(M=$ $6.47 ; \mathrm{SD}=1.23$ ). Age had no impact on the results.

\section{The working together of motive incongruence and emotional disclosure}

Participants' somatization symptoms were analysed with a hierarchical regression analysis. Using the same procedure as in Study 1, motive incongruence and emotional disclosure were entered as Step 1 and their multiplicative interaction as Step 2 in the regression equation.

The results show that neither the main effect of motive incongruence nor the main effect of emotional disclosure reached significance. Instead, their interaction significantly predicted somatization $\left(b=-0.20, s e_{\mathrm{b}}=-0.20, \Delta R^{2}=0.04, t(98)=\right.$ $-2.03, p<0.05$; overall model: $R^{2}=0.05 ; F(3$, $98)=1.61$ ). Figure 2 illustrates that participants who have a high implicit-explicit motive incongruence and who additionally did not share their emotions with their partner reported the most somatization symptoms. Within participants with low motive incongruence, it made no difference whether they disclose emotions or not. Post hoc analyses confirmed that the difference between participants with high and low amounts of emotional disclosure was marginally significant for

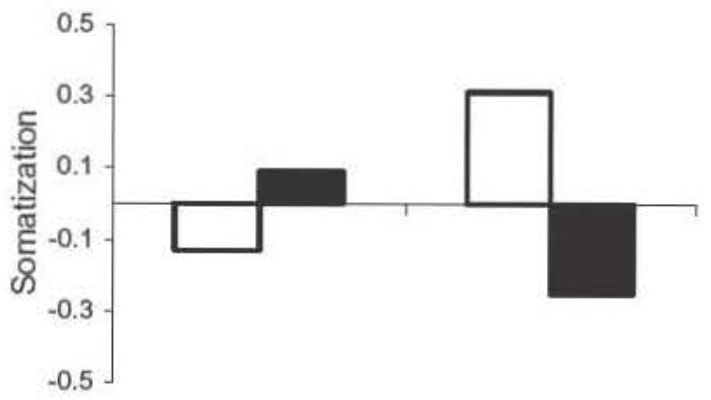

incongruence low incongruence high

DDisclosure low Disclosure high

Figure 2. Somatization as a function of motive incongruence and emotional disclosure (Study 2). motive incongruent participants ${ }^{2}(t(98)=-1.98$, $p=0.051$, two-tailed test), but not significant for motive congruent participants, $t(98)=0.82, p=$ 0.41 , two-tailed test).

\section{Brief discussion}

Study 2 could replicate the moderator effect found in Study 1 with different operationalizations of the implicit (TAT vs. OMT) and the explicit motivational system (explicit motives vs. goals), and thus for motive incongruence. Participants with a high incongruence between their implicit and explicit affiliation motives who practiced emotional disclosure reported less somatization symptoms compared to participants with a high incongruence without disclosing their emotions.

\section{General discussion}

Present research explained the negative effects of motive incongruence on health by assuming that a chronically suppressed implicit motive or an explicit motive (or goal) that lacks the energy of an implicit motive both function as a chronic source of stress. This chronic stressor is assumed to result in an enduring high level of the stress hormone cortisol, which again impairs long-term health. Referring to a broad empirical basis that emotional disclosure effectively reduces stress (see Smyth, 1998) we concluded that it may be a suitable method to buffer the negative effects of motive incongruence on health impairment. Taking the affiliation motive as an example, we tested the hypothesis that motive incongruent individuals who use emotional disclosure would report less health impairment than motiveincongruent individuals who do not disclose their emotions.

The results of two studies confirmed this hypothesis. In Study 1, affiliation motive incongruent participants (here: incongruence between implicit motive and goals) reported less impairment of health measured by the amount of

\footnotetext{
${ }^{2}$ Motive-congruent participants are those with 1 $\mathrm{SD}$ below the mean of the motive incongruence variable; motive incongruent participants are those with $1 \mathrm{SD}$ above the mean of the motive incongruence variable.
} 
medicine intake, when they applied emotional disclosure as a stress-reducing strategy. This pattern of results was replicated in Study 2 indicating that emotional disclosure reduces the negative effect that affiliation motive incongruence (here measured by implicit motive and explicit motive) had on somatization symptoms. Interestingly, in both studies motive incongruent participants with emotional disclosure reported as less medicine intake and as less somatization symptoms, respectively, as motive congruent participants did. This indicates that emotional disclosure could nearly fully annul the negative effects of motive incongruence on health. However, the present research is limited in so far that we focused only on two aspects of health (medicine intake and somatization symptoms) and these were measured by participants' selfreports. It would be interesting to consider other and also more complex health outcomes in future studies that may also be measured by objective means (e.g. physiological variables such as cortisol level).

Present research focused on talking about emotional experiences because we wanted to examine the most naturally occurring form of emotional disclosure. The results showed that some individuals naturally used the stressreducing method of emotional disclosure through talking. But the results also indicate that some individuals did not use it and were therefore fully exposed to the negative effects of motive incongruence. For them, an intervention would be useful in which emotional disclosure is artificially induced. The success that Pennebaker achieves with his emotional disclosure writing paradigm (Pennebaker \& Beall, 1986; Pennebaker et al., 2001; Smyth, 1998) encourages applying the paradigm on motive incongruent participants and testing whether it helps them to improve their well-being.

The results of present research are of theoretical interest for stress research as well as for motive-incongruence research. It is common sense in the psychology- and medicine-based stress research that not only situational stress, but also intrapersonal stress, causes impairment of subjective well-being and health. It has also been shown that this intrapersonal stress has not necessarily be the consequence of severe traumatic experiences (e.g. accidents, death of a beloved), but can also be everyday stress, such as worries about jobs and relationships. Present research introduces another everyday stressor to this approach, which is motive incongruence. Being chronically dissatisfied because one's implicit motives are not satisfied due to non-matching explicit motives and goals is an important source of stress in everyday life. Additionally, striving for explicit goals and motives that are not energized by an implicit motive and therefore require a high amount of volitional control is mentally exhausting and also causes intrapersonal stress. Present research showed that a stress-reducing method buffers the negative effects of motive incongruence on health indicators such as medicine intake and somatization symptoms.

Motive-incongruence research has not only demonstrated the negative effects of motive incongruence on well-being, but also has suggested a method to deal with it. Schultheiss and Brunsten (1999) reasoned that a way to enhance congruence between implicit motive and goals is to test whether a potential goal fits to one's implicit motives before pursuing the goal. Because the emotion-based implicit motives are unconscious, and therefore can not be compared directly with the conscious cognition-based motives, the authors suggested a translation process bridging the gap between the two types of motives. They suggested and empirically supported goal imagery, the "perception-like mental representation of the pursuit and attainment of a goal' (Schultheiss \& Brunstein, 1999, p. 5) as a method to link both motivational systems.

Whereas Schultheiss and Brunstein's (1999) method can be described as a preventive method, because goal imagery a priori prevents chronic stress by reducing motive incongruence, the method applied in present research is better described as a curative method. Emotional disclosure fights the negative effects of chronic stress that is already aroused as a result of motive incongruence. In order to examine more in detail the preventive and curative methods of dealing with motive incongruence further studies are needed. For example, it is necessary to demonstrate that the goal imagery effect holds also true for health indicators, and that the emotional disclosure effect can be replicated in longitudinal studies with subjective and objective well-being indicators as dependent variables.

Eventually, talking to others, on the long run, not only might reduce the negative affective consequences of motive incongruence, but also may reduce motive incongruence itself. As Pennebaker and Francis (1996) observed 'subjects who [...] talked about upheavals evidenced greater cogni- 
tive changes and [...] gained a better understanding of the problem' (p. 603). In the present context these cognitive changes could involve a deeper understanding of one's implicit needs and motives, on the one hand, and one's explicit goals, on the other hand. Becoming aware of incongruence, then, could instigate self-regulatory mechanisms aimed at diminishing the incongruence by, for example, looking for opportunities to act on an implicit motive, or disengaging from motive incongruent goals.

\section{Acknowledgement}

The authors thank Alice Hollenstein for her help in data collection.

\section{References}

Atkinson, J.W. (1957). Motivational determinants of risktaking behavior. Psychological Review, 64, 359-372.

Baumann, N., Kaschel, R., \& Kuhl, J. (2005). Striving for unwanted goals: Stress-dependent discrepancies between explicit and implicit achievement motives reduce subjective well-being and increase psychosomatic symptoms. Journal of Personality and Social Psychology, 89 (5), 781-799.

Baumeister, R., \& Leary, M.R. (1995). The need to belong: Desire for interpersonal attachments as a fundamental human motivation. Psychological Bulletin, 117, 497-529.

Bernard, M., Jackson, C., \& Jones, C. (2006). Written emotional disclosure following first-episode psychosis: Effects on symptoms of post-traumatic stress disorder. British Journal of Clinical Psychology, 45 (3), 403-415.

Bodenmann, G. (1990). Stress und Coping bei Paaren [Stress and coping in couples]. Göttingen: Hogrefe.

Brunstein, J.C. (1993). Personal goals and subjective wellbeing. Journal of Personality and Social Psychology, 65, 1061-1070.

Brunstein, J.C., Lautenschläger, U., Nawroth, B., Pöhlmann, K., \& Schultheiss, O. (1995). Persönliche Anliegen, soziale Motive und emotionales Wohbefinden [Personal concerns, social motives, and emotional well-being]. Zeitschrift für Differentielle und Diagnostische Psychologie, 16, 1-10.

Brunstein, J.C., Schultheiss, O.C., \& Grässmann, R. (1998). Personal goals and emotional well-being: The moderating role of motive dispositions. Journal of Personality and Social Psychology, 75, 2, 494-508.

Brunstein, J.C., Schultheiss, O.C., \& Maier, G.W. (1999). The pursuit of personal goals. In J. Brandtstädter, \& R.M. Lerner (Eds), Action \&o self-development (pp. 169-196). London: Sage Ltd.

Cohen, P., Cohen, J., West, S.G., \& Aiken, L.S. (2003). Applied multiple regression/correlation analysis fort he behavioral sciences (3rd edition). Hillsdale, NJ: Lawrence Erlbaum.

DeCharms, R., Morrison, H.W., Reitman, W.R., \& McClelland, D.C. (1955). Behavioral correlates of directly and indirectly measured achievement motivation. In D.C. McClelland (Ed.), Studies in motivation (pp. 414-423). New York: Appleton-Century-Crofts.
deVicente, A., Munoz, M., Perez, S.E., \& Santos-Olmo, A.B. (2004). Emotional disclosure in homeless people: A pilot study. Journal of Traumatic Stress, 17 (5), 439-443.

Dormann, C., \& Zapf, D. (2004). Customer-related social stressors and burnout. Journal of Occupational Health Psychology, 9 (1), 61-82.

Elliot, A.J., Gable, S.L., \& Mapes, R.R. (2006). Approach and avoidance motivation in the social domain. Personality and Social Psychology Bulletin, 32 (3), 378-391.

Epstein, S. (1994). Integration of the cognitive and the psychodynamic unconscious. American Psychologist, 49, 709724.

Francis, M.E., \& Pennebaker, J.W. (1992). Putting stress into words: The impact of writing on physiological, absentee, and self-reported emotional well-being measures. American Journal of Health Promotion, 6, 280-287.

Franke, G.H. (1994). Eine weitere Überprüfung der Symptomcheckliste (SCL-90-R) als Forschungsinstrument [Further assessment of the Symptom Check List (SCL-90-R) as a research instrument]. Diagnostica, 38 (2), 160-167.

Frattaroli, J. (2006). Experimental Disclosure and its moderators: A meta-analysis. Psychological Bulletin, 132 (6), 823-865.

Göhring, S. (2002). Zur Messung des Konstrukts Selbstenthüllung unter besonderer Berücksichtigung der evolutionistischen Emotionstheorie. Frankfurt am Main: Peter Lang.

Greenberg, M.A., Wortmann, C.B., \& Stone, A.A. (1996). Emotional expression and physical health: Revising traumatic memories for fostering self-regulation. Journal of Personality and Social Psychology, 71, 588-602.

Grulke, N., Bailer, H., Blaser, G., Geyer, M., Schmutzer, G., Brähler, E., \& Albani, C. (2006). Worrying about one's job, family, financial situation, and health-results of a population-representative study. Psycho-Social-Medicine, 3, 110.

Heckhausen, H. (1989). Motivation und Handeln [Motivation and Action]. Berlin: Springer.

Hofer, J., \& Chasiotis, A. (2003). Congruence of life goals and implicit motives as predictors of life satisfaction: Crosscultural implications of a study of Zambian male adolescents. Motivation and Emotion, 27, 3, 251-272.

Hofer, J., Chasiotis, A., \& Campos, D. (2006). Congruence between social values and implicit motives: Effects on life satisfaction across three cultures. European Journal of Personality, 20, 305-324.

Jackson, D.N. (1984). Personality Research Form manual. Port Huron, MI: Research Psychologists Press.

Kasser, T., \& Ryan, R.M. (1996). Further examining the American dream: Differential correlates of intrinsic and extrinsic goals. Personality and Social Psychology Bulletin, $22,80-87$.

Kehr, H.M. (2004). Implicit/explicit motive discrepancies and volitional depletion among managers. Personality and Social Psychology Bulletin, 30, 3, 315-327.

Kelley, J.E., Lumley, M.A., \& Leisen, J.C. (1997). Health effects of emotional disclosure in rheumatoid arthritis patients. Health Psychology, 16, 331-340.

Kiselica, M.S., Baker, S.B., Thomas, R.N., \& Reedy, S. (1994). Effects of stress inoculation training on anxiety, stress, and academic performance among adolescents. Journal of Counseling Psychology, 41, 335-342.

Kuhl, J., \& Scheffer, D. (1999). Der operante Multi-MotiveTest (OMT): Manual [The operant multi-motive-test (OMT): Manual]. University of Osnabrück, Germany.

Langens, T.A., \& Schüler, J. (2005). Written emotional expression and emotional well-being: The moderating role 
of fear of rejection. Personality and Social Psychology Bulletin, 31, 818-830.

Lee, C., \& Gramotnev, H. (2007). Life transitions and mental health in a national cohort of young Australian women. Developmental Psychology, 43 (4), 877-888.

Lepore, S.J. (1997). Social-environmental influences on the chronic stress process. In: Gottlieb, B. (Ed.), Coping with chronic stressors (pp. 133-160). New York: Plenum.

Lepore, S.J., \& Greenberg, M.A. (2002). Mending broken hearts: Effects of expressive writing on mood, cognitive processing, social adjustment and health following a relationship breakup. Psychology and Health, 17, 547560.

Lepore, S.J., \& Smyth, J.M. (2002). Writing cure: How expressive writing promotes health and emotional well-being. Washington, DC: American Psychological Association.

Lepore, S.J., Greenberg, M.A., Bruno, M., \& Smyth, J.M. (2002). Expressive writing and health: Self-regulation of emotion-related experience, physiology, and behaviour. In S.J. Lepore, \& J. Smyth (Eds), The writing cure: How expressive writing influences health and well-being, (pp. 199-232). American Psychological Association, Washington, DC.

McAdams, D.P., \& Bryant, F.B. (1987). Intimacy motivation and subjective mental health in a nationwide sample. Journal of Personality, 55, 395-413.

McClelland, D.C. (1985). Human motivation. Glenview, IL: Scott, Foresman.

McClelland, D.C. (1991). The Personal Value Questionnaire. Boston: McBer \& Co.

McClelland, D.C., \& Jemmott, J.B. (1980). Power motivation, stress, and physical illness. Journal of Human Stress, 6, 6-15.

McClelland, D.C., Koestner, R., \& Weinberger, J. (1989). How do self-attributed and implicit motives differ? Psychological Review, 96, 4, 690-702.

McGuire, K.M.B., Greenberg, M.A., \& Gevirtz, R. (2005). Autonomic effects of expressive writing in individuals with elevated blood pressure. Journal of Health Psychology, 10, 197-207.

Mendolia, M., \& Kleck, R.E. (1993). Effects of talking about a stressful event on arousal: Does what we talk about make a difference? Journal of Personality and Social Psychology, 64, 283-292.

Murray, H.A. (1938). Explorations in personality. New York: Wiley.

Murray, H.A. (1943). The Thematic Apperception Test: Manual. Cambridge, MA: Harvard University Press.

Murray, E.J., \& Segal, D.L. (1994). Emotional processing in vocal and written expression of feelings about traumatic experiences. Journal of Traumatic Stress, 7, 391-405.

Norman, S.A., Lumley, M.A., Dooley, J.A., \& Diamond, M. P. (2004). For whom does it work? Moderators of the effects of written emotional disclosure in a randomized trial among women with chronic pelvic pain. Psychosomatic Medicine, 66 (2), 174-183.

Panagopoulou, E., Maes, S., Rimé, B., \& Montgomery, A. (2006). Social sharing of emotion in anticipation of cardiac surgery: Effects on preoperative distress. Journal of Health Psychology, 11, 809-820.

Park, C.L., \& Blumberg, C.J. (2002). Disclosing trauma through writing: Testing the meaning-making hypothesis. Cognitive Therapy and Research, 26, 597-616.

Parkinson, B., \& Totterdell, P. (1999). Classifying affectregulation strategies. Cognition and Emotion, 13, $277-$ 303.
Pennebaker, J.W. (1997). Opening up: The healing power of expressing emotions (Revised edition). New York: Guilford Press.

Pennebaker, J.W., \& Beall, S. (1986). Confronting a traumatic event: Toward an understanding of inhibition and disease. Journal of Abnormal Psychology, 95, 274281.

Pennebaker, J.W., \& Francis, M.E. (1996). Cognitive, emotional, and language processes in disclosure. Cognition and Emotion, 10, 601-626.

Pennebaker; J.W., \& Seagal, J.D. (1999). Forming a story: The health benefits of narrative. Journal of Clinical Psychology, 55, 1243-1254.

Pennebaker, J.W., Colder, M., \& Sharp, L.K. (1990). Acceleration the coping process. Journal of Personality and Social Psychology, 58, 528-537.

Pennebaker, J.W., Mayne, T.J., \& Francis, M.E. (1997). Linguistic predictions of adaptive bereavement. Journal of Personality and Social Psychology, 72, 863-871.

Pennebaker, J.W., Zech, E., \& Rimé, B. (2001). Disclosing and sharing emotion: psychological, social and health consequences. In M.S. Stroebe, R.O. Hansson, W. Stroebe, \& H. Schut (Eds). Handbook of bereavement research: Consequences, coping, and care (pp. 517-543). Washington, DC: American Psychological Association.

Petrie, K.J., Booth, R.J., Pennebaker, J.W., Davison, K P., \& Thomas, M.G. (1995). Disclosure of trauma and immune response to a hepatitis $B$ vaccination program. Journal of Consulting and Clinical Psychology, 63, 787792.

Rimé, B. (1987). Le partage social des émotions [Social sharing of emotions]. Paper presented at the Symposium on Social Psychology and the Emotions, Maison des Sciences de l'Homme, Paris.

Rimé, B. (1999). Expressing emotion, physical health, and emotional relief: A cognitive-social perspective. Advances in Mind-Body Medicine, 15, 175-179.

Rimé, B., Philippot, P., Boca, S., \& Mesquita, B. (1992). Long-lasting cognitive and social consequences of emotion: Social sharing and rumination. In W. Stroebe, \& M. Hewstone (Eds), European review of social psychology (Vol. 3, pp. 225-258). Chichester, UK: John Wiley \& Sons.

Roxburgh (2004). 'There just aren't enough hours in the day'. The mental-health concequences of time-pressure. Journal of Health and Social Behavior, 45, 115-131.

Ryan, R.M., Chirkov, V.I., Little, T.D., Sheldon, K.M., Tomoshina, E., \& Deci, E.L. (1999). The American dream in Russia: Extrinsic aspirations and well-being in two cultures. Personality and Social Psychology Bulletin, 25, 1501524.

Sapolski, R.M. (1992). Stress, the aging brain, and the mechanism of neuron death. Cambridge, MA: MIT Press.

Scheffer, D. (2005). Implizite Motive [Implicit motives]. Göttingen: Hogrefe.

Scheffer, D., Kuhl, J., \& Eichstaedt, J. (2003). Der Operante Motiv-Test (OMT): Inhaltsklassen, Auswertung, psychometrische Kennwerte und Validierung. [The operant motivetest (OMT): Contents, scoring, psychometric values, and validation], In J. Stiensmeier-Pelster, \& F. Rheinberg (Eds), Diagnostik von Motivation und Selbstkonzept [Diagnostic of motivation and self-concept] (pp. 151-1167). Göttingen: Hogrefe.

Schultheiss, O.C., \& Brunstein, J. C. (1999). Goal imagery: Bridging the gap between implicit motives and explicit goals. Journal of Personality, 67, 1-38. 
Sheldon, K.M., \& Elliot, A.J. (1999). Goal striving, need satisfaction, and longitudinal well-being: The self-concordance model. Journal of Personality and Social Psychology, 76, 482-497.

Sheldon, K.M., \& Kasser, T. (1995). Coherence and congruence: Two aspects of personality integration. Journal of Personality and Social Psychology, 68, 531-543.

Sloan, D.M., \& Marx, B.P. (2004). Taking pen to hand: Evaluating theories underlying the written disclosure paradigm. Clinical Psychology: Science and Practice, 11, 121137.

Sloan, D.M., Marx, B P., \& Epstein, E.M. (2005). Further examination of the exposure model underlying the efficacy of written emotional disclosure. Journal of Consulting and Clinical Psychology, 73 (3), 549-554.

Smith, C P. (1992). Motivation and personality: Handbook of thematic content analysis. Cambridge: Cambridge University Press.

Smith, C P., Feld, S.C., \& Franz. C.E. (1992). Methodological considerations: steps in research employing content analysis systems. In C P. Smith (Ed.), Motivation and personality: Handbook of thematic content analysis (pp. 515-536). Cambridge, MA: Cambridge University Press.
Smyth, J.M. (1998). Written emotional expression: Effect sizes, outcome types, and moderating variables. Journal of Consulting and Clinical Psychology, 66, 174-184.

Sokolowski, K. (2008). Social bonding: Affiliation motivation and intimacy motivation. In J. Heckhausen, \& $\mathrm{H}$. Heckhausen (Eds), Motivation and action (pp. 184-201), Cambridge, MA: Cambridge University Press.

Sokolowski, K., Schmalt, H.-D., Langens, T.A., \& Puca, R. M. (2000). Assessing achievement, affiliation, and power motives all at once-The Multi-Motive Grid (MMG). Journal of Personality Assessment, 74, 126-145.

Story, L.B., \& Repetti, R. (2006). Daily occupational stressors and marital behavior. Journal of Family Psychology, 20 (4), 690-700.

Winter, D.G. (1991). Manual for scoring motive imagery in running test (3rd ed.). Unpublished scoring manual. University of Michigan.

Winter, D.G. (1996). Personality: Analysis and interpretation of lives. New York: McGraw-Hill.

Zakowski, S.G., Ramati, A., Morton, C., Johnston, P., \& Flanigan, R. (2004).Written emotional disclosure buffers the effects of social constraints on distress among cancer patients. Health Psychology, 23 (6), 555-563. 